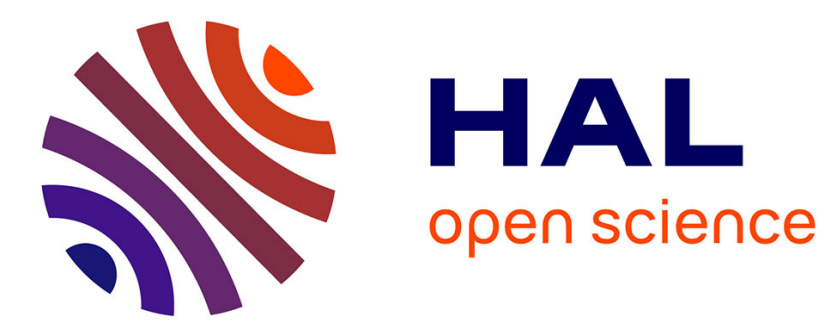

\title{
Density of states of quasiparticles in an anisotropic flat slab
}

\author{
Ya. A. Iosilevskii
}

\section{To cite this version:}

Ya. A. Iosilevskii. Density of states of quasiparticles in an anisotropic flat slab. Journal de Physique Lettres, 1979, 40 (1), pp.11-14. 10.1051/jphyslet:0197900400101100 . jpa-00231554

\section{HAL Id: jpa-00231554 https://hal.science/jpa-00231554}

Submitted on 1 Jan 1979

HAL is a multi-disciplinary open access archive for the deposit and dissemination of scientific research documents, whether they are published or not. The documents may come from teaching and research institutions in France or abroad, or from public or private research centers.
L'archive ouverte pluridisciplinaire HAL, est destinée au dépôt et à la diffusion de documents scientifiques de niveau recherche, publiés ou non, émanant des établissements d'enseignement et de recherche français ou étrangers, des laboratoires publics ou privés. 


\title{
Density of states of quasiparticles in an anisotropic flat slab
}

\author{
Ya. A. Iosilevskii \\ Department of Physics, Technion \\ Israel Institute of Technology, Haifa 32000, Israel. \\ (Reçu le 27 juillet 1978, révisé le 8 novembre 1978, accepté le 13 novembre 1978)
}

\begin{abstract}
Résumé. - La densité d'états de quasiparticules dans une plaque anisotrope ou dans un demi-espace est calculée dans l'approximation du tenseur de masse effective. Les résultats ainsi obtenus sont utilisés pour évaluer la contribution de la surface à la densité d'états ; cette contribution est proportionnelle au rapport de la surface au volume. Sous certaines conditions discutées dans l'article, la contribution de surface trouvée reste valable pour un ensemble de petites particules conductrices de forme arbitraire.
\end{abstract}

Abstract. - The density of states of quasiparticles in an anisotropic flat slab or half-space is calculated in the effective mass tensor approximation. The results obtained are used to find the surface contribution to the density of states which is proportional to the surface-to-volume ratio. Under certain conditions discussed in the paper, the surface contribution found remains valid for an assembly of small conducting particles of arbitrary shape.

1. Introduction. - The purpose of this paper is to obtain the density of states, $g(E)$, of an arbitrary scalar field in an anisotropic flat slab with rigid boundaries. We proceed from the general relation

$g(E)= \pm(\pi V)^{-1} \lim _{\gamma \rightarrow \mp 0} \int_{V} \operatorname{Im} D(\mathbf{r}, \mathbf{r} ; E+i \gamma) \mathrm{d}^{3} r$,

which is valid for any finite sample (homogeneous or inhomogeneous) of volume $V . D\left(\mathbf{r}, \mathbf{r}_{0} ; w\right)$ is the Green's function of the field in the sample; $w$ is an energy-like complex parameter; $E$ is the real energy of a state of the field. Both choices of signs in eq. (1) (two upper or two lower) are equivalent to each other. Since the real part of $D\left(\mathbf{r}, \mathbf{r}_{0} ; w\right)$ diverges at $\mathbf{r}=\mathbf{r}_{0}$, the substitution $\mathbf{r}=\mathbf{r}_{0}$ and the integration with respect to $\mathbf{r}$ must be performed in eq. (1) after taking the imaginary part. In general, by the word sample we mean all that region of space which is penetrated by the field. We denote the volume of this region by $V$. By this definition, we are able to avoid entering into the complicated problem of self-consistency for charged particles discussed by Stratton [1].

In the case under consideration, the Green's function is the solution of the equation

$\left(w+\varepsilon^{i k} \nabla^{i} \nabla^{k}\right) D\left(\mathbf{r}, \mathbf{r}_{0} ; w\right)=\delta\left(\mathbf{r}-\mathbf{r}_{0}\right)$,

$x, x_{0} \in\left(d_{0}, d_{1}\right)$, subject to

$\left.D\left(\mathbf{r}, \mathbf{r}_{0} ; w\right)\right|_{x=d_{0}}=\left.D\left(\mathbf{r}, \mathbf{r}_{0} ; w\right)\right|_{x=d_{1}}=0$.

The latin superscripts denote projections onto the axes of a Cartesian coordinate system. For convenience, the $x$-axis is aligned with a normal to the boundary planes. All repeated superscripts are summed from 1 to 3 . The quantity $D\left(\mathbf{r}, \mathbf{r}_{0} ; w\right)$ satisfying eqs. (2) and (3) was found by a modified method of images in reference [2] and by the Dyson's equations method in reference [3].

In eq. $(2), \varepsilon^{i k}$ is a material tensor $\left(\varepsilon^{i k}=\varepsilon^{k i}\right)$, which specifies the nature of the field. Without loss of generality, we can put

$\varepsilon^{i k}=\frac{1}{2} \hbar^{2}\left(\hat{m}^{-1}\right)^{i k}$

As a consequence, any field of the class under consideration proves to be equivalent to the wave field of a quasiparticle with the effective mass tensor $m^{i k}$ and the total energy $w$ (in the general case, $w$ is complex), which moves in a constant potential $(U=0)$ within the given sample.

In contrast to the approach of this paper, where the total density of states (the trace of the spectral density of the Green's function) is calculated in a macroscopic approximation, many calculations, mainly of the local density of electron states (the spectral density of the 
corresponding Green's function for coinciding spatial arguments) near the plane surface of a half-space, have been made previously (see, e.g. Refs. [4-6]) in the framework of some microscopic models of a crystal and its surface. (The difference between the total and local densities of states of any quasiparticles is essentially the same as in the case of phonons [7].)

Having calculated $g(E)$ for a flat slab, we can establish conditions under which the surface contribution to $g(E)$ is additive, i.e. proportional to the surface-to-volume ratio, and find this contribution explicitly. In this way, we can find the effect of surfaces on the density of states and on electronic properties of an assembly of small conducting particles of an arbitrary shape (cf. a similar problem for acoustic phonons [8]). A statistical approach to such a problem was developed in references $[9,10]$.

2. Solution of the problem. - In the case of a flat slab, the Green's function can be written in the form of Fourier analysis

$$
\begin{aligned}
& D\left(\mathbf{r}, \mathbf{r}_{0} ; w\right)=(2 \pi)^{-2} \int D\left(x, x_{0} ; \mathbf{f}_{\|}, w\right) \times \\
& \quad \times \exp \left(i \mathbf{f}_{\|} \cdot\left(\mathbf{r}_{\|}-\mathbf{r}_{0 \|}\right)\right) \mathrm{d}^{2} f_{\|} .
\end{aligned}
$$

The mark // stands for the orthogonal projection of a vector onto the $(y, z)$-plane, so that $\mathbf{r}_{\|}=(0, y, z)$ and $\mathbf{f}_{\|}=\left(0, f^{y}, f^{z}\right)$.

We insert eq. (5) into eq. (1) and choose the integration region $V$ in the form of a right cylinder or prism (in particular, it may be a rectangular parallelepiped) with arbitrary bases of an area $S$ situated in the boundary planes $x=d_{0}$ and $x=d_{1}$. As a result, eq. (1) becomes

$$
\begin{aligned}
& g(E)= \pm \frac{1}{\pi(2 \pi)^{2} l} \times \\
& \quad \times \lim _{\gamma \rightarrow \mp 0} \int \mathrm{d}^{2} f_{\|} \int_{d_{0}}^{d_{1}} \mathrm{~d} x \operatorname{Im} D\left(x, x ; \mathbf{f}_{\|}, E+i \gamma\right),
\end{aligned}
$$

where $l=d_{1}-d_{0}$ is the thickness of the slab.

According to reference [2], we have

$$
D\left(x, x ; \mathbf{f}_{\|}, w\right)=D_{0}\left(0 ; \mathbf{f}_{\|}, w\right)+\Delta D\left(x, x ; \mathbf{f}_{\|}, w\right),
$$

where

$$
\begin{aligned}
& D_{0}\left(x ; \mathbf{f}_{\|}, w\right)=(2 \pi)^{-1} \int_{-\infty}^{\infty}\left(w-\varepsilon^{i k} f^{i} f^{k}\right)^{-1} \times \\
& \quad \times \exp \left(i f^{x} x\right) \mathrm{d} f^{x}=-\left(2 \varepsilon^{x x} q\right)^{-1} \exp (i p x-q|x|),
\end{aligned}
$$

$$
\begin{gathered}
\Delta D\left(x, x ; \mathbf{f}_{\|}, w\right)=\left(4 \varepsilon^{x x} q \sinh q l\right)^{-1} \exp (-q l) \times \\
\quad \times\left\{\exp \left[2 q\left(x-d_{0}\right)\right]+\exp \left[2 q\left(d_{1}-x\right)\right]-2\right\}
\end{gathered}
$$

and

$$
\begin{aligned}
& p=-\left(\varepsilon^{x x}\right)^{-1} \varepsilon^{x k} f_{\|}^{k}, \quad q=\left(\varepsilon^{x x}\right)^{-1 / 2}(u-w)^{1 / 2}, \\
& u=\lambda_{\|}^{i k} f_{\|}^{i} f_{\|}^{k}>0 ; \\
& \lambda_{\|}^{i k}=\varepsilon^{i k}-\left(\varepsilon^{x x}\right)^{-1} \varepsilon^{x i} \varepsilon^{x k}, \quad\{i, k\}=\{y, z\} ;
\end{aligned}
$$

$\operatorname{Re} q>0, \quad\left(\varepsilon^{x x}\right)^{1 / 2}>0$

(see eqs. (26)-(29) of Ref. [2]). The quantity $D_{0}\left(x ; \mathbf{f}_{\|}, w\right)$, eq. (8), is the Green's function of an infinite medium in the $\left(x, \mathbf{f}_{\|}\right)$-representation [8]. The quadratic form $\underline{u}$ is supposed to be positively definite.

Inserting eqs. (7)-(9) into eq. (6) and integrating with respect to $x$, we are left with the result

$g(E)=g^{(v)}(E)+g^{(s)}(E)$,

where

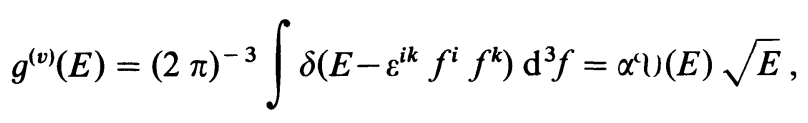

$\alpha=(2 \pi)^{-2}(\operatorname{det} \hat{\varepsilon})^{-1 / 2}=\left(2^{1 / 2} \pi^{2} \hbar^{3}\right)^{-1}(\operatorname{det} \hat{m})^{1 / 2}$,

and

$g^{(s)}(E)= \pm(\pi l)^{-1}(2 \pi)^{-2} \times$

$$
\times \lim _{\gamma \rightarrow \mp 0} \int \operatorname{Im}[F(q)]_{w=E+i \gamma} \mathrm{d}^{2} f_{\|},
$$

$F(q)=\frac{\partial}{\partial w} \ln \left[q^{-1} \sinh (q l) \exp (-q l)\right]$.

The unit step function $\mathcal{U}(E)$ appears in eq. (12) since we suppose that the quadratic form $\varepsilon^{i k} f^{i} f^{k}$ is positively definite. The integral in this equation is readily calculated if we introduce new variables of integration $s^{j}(j=1,2,3)$ by the transformation $f^{i}=\left(\hat{\varepsilon}^{-1 / 2}\right)^{i j} s^{j}$ and take into account that the Jacobian of this transformation is $\operatorname{det} \hat{\varepsilon}^{-1 / 2}=(\operatorname{det} \hat{\varepsilon})^{-1 / 2}$. In writing the final expression for $\alpha$ in eq. (13), we have made use of eq. (4). The quantity $g^{(v)}(E)$ as given by eqs. (12) and (13) is the usual bulk density of electron states in the effective mass tensor approximation. At the same time, the quantity $g^{(s)}(E)$ defined by eqs. (14) and (15) is the contribution to $g(E)$ from the boundary planes. The final result in eq. (15) can be verified if we take into account that $(\partial q / \partial w)=-\left(2 \varepsilon^{x x} q\right)^{-1}$ (see the definition of $q$, eqs. (10)).

In order to calculate $g^{(s)}(E)$, we introduce new variables of integration $s_{\|}^{j}(j=2,3)$ by putting

$f_{\|}^{i}=\sum_{j=2,3}\left(\hat{\lambda}_{\|}^{-1 / 2}\right)^{i j} s_{\|}^{j}$.

As a result, eq. (14) becomes

$g^{(s)}(E)=(4 \pi l)^{-1} J \varphi(E)$, 
where

$$
\begin{aligned}
J= & \left|\frac{\partial\left(f_{\|}^{2}, f_{\|}^{3}\right)}{\partial\left(s_{\|}^{2}, s_{\|}^{3}\right)}\right|=\operatorname{det} \hat{\lambda}_{\|}^{-1 / 2}=\left(\operatorname{det} \hat{\lambda}_{\|}\right)^{-1 / 2}= \\
& =\left(\varepsilon^{x x}\right)^{1 / 2}(\operatorname{det} \hat{\varepsilon})^{-1 / 2}=2 \hbar^{-2}\left[\left(\hat{m}^{-1}\right)^{x x} \operatorname{det} \hat{m}\right]^{1 / 2}
\end{aligned}
$$

is the Jacobian of the above transformation, and

$\varphi(E)= \pm \pi^{-1} \lim _{\gamma \rightarrow \mp 0} \int_{0}^{\infty} \operatorname{Im}[F(q)]_{w=E+i \gamma} \mathrm{d} u$

$u=s_{\|}^{2}$.

In developing the final result in eq. (17), we have taken into account the relation

$\operatorname{det} \hat{\lambda}_{\|}=\lambda_{\|}^{y y} \lambda_{\|}^{z z}-\left(\lambda_{\|}^{y z}\right)^{2}=\left(\varepsilon^{x x}\right)^{-1} \operatorname{det} \hat{\varepsilon}$,

which follows from the definition of $\lambda_{\|}^{i k}$, eqs. (10), and have then made use of eq. (4). In writing eq. (18), we have integrated with respect to the polar angle of the vector $\mathbf{s}_{\|}$(this gives the factor $2 \pi$ ).

In view of the relation

$\sinh z=z \prod_{k=1}^{\infty}\left(1+\frac{z^{2}}{\pi^{2} k^{2}}\right), \quad z=q l$,

it follows from eqs. (10) and (15) that

$$
\begin{aligned}
& \pm \pi^{-1} \lim _{\gamma \rightarrow \mp 0} \operatorname{Im}[F(q)]_{w=E+i \gamma}= \\
& \quad=\sum_{k=1}^{\infty} \delta\left(u-E+\pi^{2} k^{2} \varepsilon^{x x} l^{-2}\right)+ \\
& \quad+ฯ(E-u) l\left[2 \pi \sqrt{\varepsilon^{x x}(E-u)}\right]^{-1} .
\end{aligned}
$$

In writing the last term on the right-hand side of eq. (21), we have taken into account that the quantity $\lim _{\gamma \rightarrow+}(v-i \gamma)^{1 / 2}$, subject to $\operatorname{Re}(v-i \gamma)^{1 / 2}>0$, is $\gamma \rightarrow+0$

equal to $\sqrt{v}$ if $v>0$, and to

$-i(\operatorname{sgn} \gamma) \sqrt{|v|}(1+i \gamma / 2|v|)$ if $v<0(\gamma \rightarrow \mp 0)$.

Substitution of eq. (21) into eq. (18) gives

$\varphi(E)=\psi(\xi), \quad \psi(\xi)=[\xi]-\xi$,

$\xi=\frac{l}{\pi} \sqrt{\frac{E}{\varepsilon^{x x}}}=\frac{l}{\pi \hbar} \sqrt{\frac{2 E}{\left(\hat{m}^{-1}\right)^{x x}}}$,

where $[\xi]$ stands for the integer part of $\xi$.

$\psi(\xi)$ is a universal periodic function of $\xi$ with the period $\delta \xi=1 . \varphi(E)$ is therefore a quasiperiodic function of $E$. Its period

$\delta E=(\pi \hbar / l) \sqrt{2\left(\hat{m}^{-1}\right)^{x x} E}$

slowly varies with $E$. Eq. (23) can be derived from the relation
$\Delta \xi=l\left[\pi \hbar \sqrt{2\left(\hat{m}^{-1}\right)^{x x} E}\right]^{-1} \Delta E$

by setting $\Delta E=\delta E$ and $\Delta \xi=\delta \xi=1$.

Averaging $\varphi(E)$ over a small interval $\Delta E$, we have

$$
\begin{aligned}
& \frac{1}{\Delta E} \int_{E-\Delta E / 2}^{E+\Delta E / 2} \varphi\left(E^{\prime}\right) \mathrm{d} E^{\prime} \equiv \frac{1}{\Delta \xi} \times \\
& \quad \times \int_{\xi-\Delta \xi / 2}^{\xi+\Delta \xi / 2} \psi\left(\xi^{\prime}\right) \mathrm{d} \xi^{\prime}=-\frac{1}{2}+\mathrm{O}\left(\frac{1}{\Delta \xi}\right),
\end{aligned}
$$

where, by assumption,

$\delta E \ll \Delta E \ll E, \quad 1 \ll \Delta \xi \ll \frac{l}{\pi \hbar} \sqrt{\frac{E}{2\left(\hat{m}^{-1}\right)^{x x}}}$.

$\Delta E$ and $\Delta \xi$ are connected by eq. (24). In developing the final result in eqs. (25), we have put

$\mathrm{d} E^{\prime}=2 \sqrt{E^{\prime}} \mathrm{d} \sqrt{E^{\prime}}$

and analogously $\Delta E=2 \sqrt{E} \Delta \sqrt{E}$. The quantity $\sqrt{E^{\prime}}$ remains practically unchanged over the interval $\Delta E$ and can therefore be taken out of the integral and set equal to $\sqrt{E}$.

From eqs. (16) and (25), it follows that

$g_{*}^{(s)}(E) \equiv \frac{1}{\Delta E} \int_{E-\Delta E / 2}^{E+\Delta E / 2} g^{(s)}\left(E^{\prime}\right) \mathrm{d} E^{\prime}=-\frac{S_{\text {tot }} J}{16 \pi V}$,

which is a constant independent of $E$ and proportional to the total area of the sample, $S_{\mathrm{tot}}=2 S(S=V / l)$. The quantity $J$ is given by eq. (16). For an isotropic medium, $J=2 \mathrm{~m} / \hbar^{2}$.

3. Discussion. - Eq. (27) can alternatively be obtained if we calculate $g(E)$ for a half-space (e.g. $x>0)$ with a rigid boundary $(x=0)$. In this case, eqs. (7), (8), and (10) keep their form, while [2, 3]

$\Delta D(x, x) \equiv \Delta D\left(x, x ; \mathbf{f}_{\|}, w\right)=\left(2 \varepsilon^{x x} q\right)^{-1} \exp (-2 q x)$, $x>0$.

In eq. (6), we should have put $d_{1}=\infty\left(d_{0}=0\right)$. But this would give $g(E)=g^{(v)}(E)$. In order to avoid this trivial result and find the surface contribution to the density of states, we choose $d_{1}$ to be finite but so large that $d_{1} \gg l^{(\mathrm{e})}$, where $l^{(\mathrm{e})}=(\hbar /|\gamma|) \sqrt{2 E / m}$, and $m$ is a characteristic mass of a particle. One can, for example, put $m=\left[\left(\hat{m}^{-1}\right)^{x x}\right]^{-1}$. Note that $\hbar /|\gamma|$ can be regarded as a lifetime of a particle and $\sqrt{2 E / m}$ as its velocity. The quantity $l^{(\mathrm{e})}$ can therefore be identified with a phenomenological mean free path of a particle.

Thus we fix $l=d_{1}=V / S$ and consider $V$ as the volume of the sample, and $S$ as the area of its boundary surface. At the same time, $\Delta D(x, x)$ is exponentially small at $x=d_{1}$ if $w=E+i \gamma$ and $d_{1} \gg l^{(\mathrm{e})}$ (see eqs. (10) and (28)). We can therefore replace the upper 
limit of integration $d_{1}$ by $\infty$ when integrating $\Delta D(x, x)$ with respect to $x$. As a result, we recover eqs. (11)-(14) and then (16)-(18) with $F(q)=\left(4 \varepsilon^{x x} q^{2}\right)^{-1}$ instead of that given by eq. (15). In this case, eq. (18) gives $\varphi(E)=-1 / 4$ and hence $g^{(s)}(E)=g_{*}^{(s)}(E)$, where $g_{*}^{(s)}(E)$ is defined by eq. (27) with the only difference that now $S_{\text {tot }}=S$. This could be expected since the area of the boundary surface of a flat slab is twice as large as that of a half-space. Note that if $l \gg l^{(\mathrm{e})}$, eq. (15) becomes $F(q)=\left(2 \varepsilon^{x x} q^{2}\right)^{-1}$.

As a rule, $g(E)$ is used for calculating quantities of the form

$\bar{A}=\int_{-\infty}^{\infty} A(E) g(E) \mathrm{d} E$,

where $A(E)$ is a certain smooth function of $E$. The specific peculiarities of the system are included in $g(E)$, while $A(E)$ characterizes the given physical property and remains the same for all systems.

Let us consider a system of small macroscopic particles. Let $l$ be a characteristic linear size of a separate grain or a characteristic radius of curvature of its boundary surface, $m$ be a characteristic mass of a quasiparticle, and $E_{A}$ be the order of magnitude of the energy interval over which $A(E)$ noticeably varies. In the case where $\bar{A}$ is a thermodynamic quantity, $E_{A}=k T$. If there exists $\Delta E$ such that

$\frac{\pi \hbar}{l \sqrt{m}} \ll \Delta \sqrt{E} \ll \sqrt{E_{A}}$

(cf. the inequalities (26)), one can, in calculating $\bar{A}$, replace $g(E)$ by an effective quantity, $g_{*}(E)$, averaged at each point over an interval $\Delta E$ as in eq. (27). From the above inequality it follows that $\lambda_{A} \ll l$, where $\lambda_{A}=2 \pi \hbar / \sqrt{2 m E_{A}}\left(\lambda_{A}=2 \pi \hbar / \sqrt{2 m k T}\right.$ is a thermal wavelength if $E_{A}=k T$ ). This means that each surface can, in calculating its contribution to $g_{*}(E)$, be regarded locally as a boundary plane of a half-space $(l \rightarrow \infty)$, and contributes to $g_{*}(E)$ additively.

In other words,

$\bar{A}=\int_{-\infty}^{\infty} A(E) g_{*}(E) \mathrm{d} E \quad$ if $\quad \lambda_{A} / l \rightarrow 0$,

$g_{*}(E)=g^{(v)}(E)+\left\langle g_{*}^{(s)}(E)\right\rangle$,

where $g^{(v)}(E)$ is given by eqs. (12) and (13), and $g_{*}^{(s)}(E)$ is defined by eq. (27) with $S_{\text {tot }}$ equal to the total surface area of all grains, and $V$ to their total volume. The brackets stand for the averaging over all possible orientations of the boundary surfaces with respect to the crystallographic axes. Note that $\operatorname{det} \hat{m}$ is an invariant, and $\left(\hat{m}^{-1}\right)^{x x}$ can be written $\left(\hat{m}^{-1}\right)^{i k} n^{i} n^{k}$, where $\mathbf{n}$ is a unit vector normal to the boundary surface. Combining eq. (27) with eq. (17) we have therefore

$$
\begin{aligned}
& \left\langle g_{*}^{(s)}(E)\right\rangle=-\frac{S_{\mathrm{tot}}(\operatorname{det} \hat{m})^{1 / 2}}{8 \pi \hbar^{2} V} \times \\
& \quad \times \frac{1}{4 \pi} \int_{0}^{2 \pi} \int_{0}^{\pi}\left[\left(\hat{m}^{-1}\right)^{i k} n^{i} n^{k}\right]^{1 / 2} \sin \vartheta \mathrm{d} \cup \mathrm{d} S,
\end{aligned}
$$

where ('), $\$$ ) are spherical coordinates of the vector $\mathbf{n}$. It is convenient to use the principal axes of the tensor $\left(\hat{m}^{-1}\right)^{i k}$ as coordinate axes. In the case of uniaxial crystals, the integral in eq. (32) is readily expressed in terms of elementary functions.

It can be seen from eq. (32) that the surface contribution to $g_{*}(E)$ is a constant independent of $E$, just as in the case of acoustic phonons [8, 11]. A more detailed analysis of the effect of surfaces and interfaces on the electronic density of states will be given elsewhere [12].

\section{References}

[1] Stratton, R., Phys. Lett. 19 (1965) 556.

[2] IosilevskiI, Ya. A., Phys. Lett. 65A (1978) 92.

[3] IosilevskiI, Ya. A., Ann. Phys. 107 (1977) 360.

[4] Kalkstein, D. and Soven, P., Surf. Sci. 26 (1971) 85.

[5] Werner, C., Schulte, F. K. and Bross, H., J. Phys. C 8 (1975) 3817.

[6] Desjonqueres, M. C. and Cyrot-Lackmann, F., J. Phys. F 5 (1975) 1368
[7] IosilevskiI, Ya. A., Phys. Status Solidi (b) 60 (1973) 39.

[8] IosilevskiI, Ya. A., Ann. Phys. 107 (1977) 360.

[9] Kubo, R., J. Phys. Soc. Japan 17 (1962) 975.

[10] Denton, R., Mühlschlegel, B. and Scalapino, D. J., Phys. Rev. Lett. 26 (1971) 707.

[11] Dupuis, M., Maso, R. and Onsager, L., J. Chem. Phys. 33 (1960) 1452.

[12] IosilevskiI, Ya. A., Phys. Rev. B 15 (1978) (to be published). 